\title{
Preliminary expression profile of cytokines in brain tissue of BALB/c mice with Angiostrongylus cantonensis infection
}

Liping $\mathrm{Yu}^{1,2}$, Xiaoying $\mathrm{Wu}^{3}$, Jie Wei ${ }^{2,3}$, Qi Liao ${ }^{4}$, Lian Xü ${ }^{2,3}$, Siqi Luo ${ }^{2,3}$, Xin Zeng ${ }^{2,3}$, Yi Zhao ${ }^{5}$, Zhiyue Lv $v^{2,3}$ and Zhongdao $\mathrm{Wu}^{2,3^{*}}$

\begin{abstract}
Background: Angiostrongylus cantonensis (A. cantonensis) infection can result in increased risk of eosinophilic meningitis. Accumulation of eosinophils and inflammation can result in the A. cantonensis infection playing an important role in brain tissue injury during this pathological process. However, underlying mechanisms regarding the transcriptomic responses during brain tissue injury caused by A. cantonensis infection are yet to be elucidated. This study is aimed at identifying some genomic and transcriptomic factors influencing the accumulation of eosinophils and inflammation in the mouse brain infected with A. cantonensis.

Methods: An infected mouse model was prepared based on our laboratory experimental process, and then the mouse brain RNA Libraries were constructed for deep Sequencing with Illumina Genome Analyzer. The raw data was processed with a bioinformatics' pipeline including Refseq genes expression analysis using cufflinks, annotation and classification of RNAs, IncRNA prediction as well as analysis of co-expression network. The analysis of Refseq data provides the measure of the presence and prevalence of transcripts from known and previously unknown genes.

Results: This study showed that Cys-Cys (CC) type chemokines such as CCL2, CCL8, CCL1, CCL24, CCL11, CCL7, CCL12 and CCL5 were elevated significantly at the late phase of infection. The up-regulation of CCL2 indicated that the worm of $A$. cantonensis had migrated into the mouse brain at an early infection phase. CCL2 could be induced in the brain injury during migration and CCL2 might play a major role in the neuropathic pain caused by $A$. cantonensis infection. The up-regulated expression of IL-4, IL-5, IL-10, and IL-13 showed Th2 cell predominance in immunopathological reactions at late infection phase in response to infection by $A$. cantonensis. These different cytokines can modulate and inhibit each other and function as a network with the specific potential to drive brain eosinophilic inflammation. The increase of ATF-3 expression at 21 dpi suggested the injury of neuronal cells at late phase of infection. 1217 new potential IncRNA were candidates of interest for further research.
\end{abstract}

Conclusions: These cytokine networks play an important role in the development of central nervous system inflammation caused by A. cantonensis infection.

Keywords: Angiostrongylus cantonensis, Eosinophil, Mouse model, Cytokine, Chemokine

\footnotetext{
*Correspondence: wuzhd@mail.sysu.edu.cn

${ }^{2}$ Department of Parasitology, Zhongshan School of Medicine, Sun Yat-sen

University, Guangzhou, China

${ }^{3}$ Key Laboratory for Tropical Diseases Control, The Ministry of Education, Sun

Yat-sen University, Guangzhou, China

Full list of author information is available at the end of the article
} 


\section{Background}

Angiostrongylus cantonensis (A. cantonensis) is a parasitic nematode that causes human angiostrongyliasis, the most common cause of human esoinophilic meningitis (EM) in Southeast Asia and the Pacific Basin. In recent years, more cases and several outbreaks of the disease have been reported in endemic regions [1-4]. The most common clinical manifestations of EM are fever, bitemporal or frontal headache, painful paresthesias, stiff neck and vomiting $[5,6]$. Previous studies have shown that the invasion of the central nervous system (CNS) by developing larvae of the parasite can cause eosinophilic inflammation [7-9]. The larvae generally remain in the CNS and eosinophil recruitment can cause neuropathological damage [10-13].

Inflammatory factors play a key role in the development of brain inflammation. Cytokines are a broad category of small proteins released by a large range of cells including chemokines, interferons, interleukins, tumor necrosis factor, growth factor and colony stimulating factor [14]. It has been shown that the CC type chemokines are involved in mediating eosinophil chemotaxis in various allergic inflammatory responses and autoimmune diseases through interaction with corresponding receptors on the surface of eosinophils [15-19]. The CC type of chemokines consists of at least 28 members (CCL1-28). Studies have shown a critical role for chemokines CCL 2, CCL 3, CCL 5, CCL 7, CCL 11, CCL 12, CCL 24 and CCL 28. Among these CCLs, CCL-11 (CSF eotaxin) has been shown to be associated with eosinophilic meningitis in angiostrongyliasis. Previous studies have demonstrated that increased levels of the (CCL 11) correlated with CSF eosinophilia in both clinical patients and animal models infected with $A$. cantonensis $[11,20]$. In our previous study, these findings have also been verified [21]. However, the involvement of chemokines and interleukins in the inflammatory processes caused by A. cantonensis infection was not completely clear. The underlying mechanism of the accumulation of eosinophils and the regulation of associated molecules has yet to be investigated. Currently there are very few reagents that are sensitive enough for early diagnosis and there are very few therapeutic drugs for early treatment. A better understanding of the inflammatory process in the CNS and associated molecular mechanisms caused by this parasite will provide some valuable insight for the development of possible novel diagnostic and therapeutic agents.

In our present study, the EM animal model with $A$. cantonensis infection was prepared using Balb/C mice, the transcriptome analysis of the mouse brain was done using RNA-seq by Illumina sequencing, the objective is to investigate the transcript dysregulation related to inflammatory processes caused by $A$. cantonensis infection.

\section{Methods}

\section{Ethics statement}

Animals were cared for in accordance with the guidelines developed by the China Council on Animal care, and all animal experiments were performed according to the procedures approved by the Animal Care and Use Committee of Guangdong Province, China.

\section{Preparation of animal infection model}

The third-stage larvae of A.cantonensis were obtained from the infected Amazonian snail (Ampullaria gigas) by digestion of the snail tissue in artificial gastric juice (0.3\% pepsin, $0.7 \% \mathrm{HCL}$ and $10 \mathrm{ml} / \mathrm{g}$ tissue) at $37{ }^{\circ} \mathrm{C}$ for $2 \mathrm{~h}$. The precipitates were then washed with distilled water, and the third-stage larvae were counted and collected by microscopy [22].

Fifteen Balb/C mice weighing 18-20 g were purchased from the centre of Experimental Animals, Sun Yet-Sen University. All animals were kept in a germ-free environment with free access to standard pellet diet and clean water. They were divided into 5 groups (infection for 2 days, 7 days, 14 days, 21 days and normal control group). All the treatment groups were infected with $30 \pm 2$ A.cantonensis by intragastric administration. Another 25 $\mathrm{Balb} / \mathrm{C}$ mice of the same standard were also divided into 5 groups to prepare samples for Q RT-PCR.

\section{Sample collection and RNA Extraction}

In this study, the mouse brain tissues were collected on the 2 nd, 7 th, 14th and 21st day post-infection. Similarly the samples from the control mice were collected on the 21 st day post infection, and the samples were frozen in liquid nitrogen. Three samples were collected separately to prepare five pools representing an infection model and control mice for constructing RNA libraries. Total RNA was extracted using Trizol reagent (Invitrogen, Carlsbad, CA, USA) according to the manufacturer's instructions. After total RNA was resuspended in DEPCtreated water, they were stored at $-80{ }^{\circ} \mathrm{C}$ until further use. The quantity and integrity of the total RNA was assessed with an Agilent 2100 Bioanalyzer (Agilent Technologies, USA).

\section{Histochemistry examination}

Samples were collected at 21 dpi (days post infection). Blood samples were drawn from the tail of the control and EM mice from 9 to 11 in the morning and used to make peripheral blood smears, followed by staining using the Wright Giemsa staining method for the detection of eosinophils. Specimens of brain tissue of these two groups were fixed in $4 \%$ paraformaldehyde for 2 days. Then they were embedded in paraffin, serially sectioned and stained with hematoxylin eosin (HE) according to the conventional staining methods [23]. 


\section{Construction of RNA libraries and deep sequencing}

The RNA libraries were constructed from five groups respectively. The overall flow of RNA library construction and deep sequencing is shown schematically in Additional file 1: Figure S1. In brief, isolation and purification of mRNA, conversion of RNA to cDNA, followed by addition of sequencing adapters. Subsequently, the ligated RNAs were used as templates for RT-PCR amplification. The DNA sequencing was performed with Illumina Genome Analyzer to produce digital-quality data after the purification of the PCR products (Additional file 1: Figure S1). Those genes that were regulated more than 2 -fold and $\mathrm{p}<0.05$ were considered as differentially expressed [24]. The expression levels of transcripts were calculated using Cufflinks, and the data was processed with $\mathrm{R}$ software.

The raw data was processed with a bioinformatics' pipeline as follows: (1) Filter low quality tags; (2) Trim adaptor; (3) Clean reads mapping to the Mus musculus 9 genome using TopHat; (4) Refseq genes expression analysis using cufflinks; (5) Annotate and classify RNAs into different categories; (6) LncRNA prediction; (7) Coexpression network (Additional file 2: Figure S2).

LncRNA prediction TopHat was used to blast the sequences with the reference genome. Then the transcripts were re-built with Cufflinks. These transcripts were compared to previously annotated genes with Cuffcompare and obtained the information about the intergenic transcript, full intron transcript and antisense transcript. These new transcripts were used to further lncRNA prediction. According to new Transcript length $>200 \mathrm{bp}$, new transcript ORF (Open Reading Frame) length $<300$ and characters of code and noncode genes of known database, noncode screening model was built to screen new lncRNA [25]. Cufflinks calculated the expression of these new lncRNA, and Cuffdiff analyzed the differential expression of them.

\section{Analysis of refseq genes Co-expression network}

After removing the transcripts (with the maximum FPKM from 5 samples $<1$ ), expression data was normalized. By applying the variance filters, the low variance (one-fourth) was excluded from the total refseq genes. Then the Pearson correlations were calculated for all pairs of refseq genes left and used as the basis for building the networks. We calculated the Fisher's asymptotic P values using WGCNA program of $\mathrm{R}$. Then the multtest of $\mathrm{R}$ was used to adjust the $\mathrm{P}$ values into $\mathrm{q}$ values with $\mathrm{BH}$ multitest adjustments. The data of the top former and latest $0.5 \%$ correlations with $\mathrm{q}<0.01$ were kept to build the refseq genes co-expression network. The hub-gene function analysis: For each non-coding gene, GO annotation for each code gene which was co-expressed with the non-coding gene (with $\mathrm{p}<0.05$, the number of co-expressed mRNA $\geq 2$ ).
Function analysis of module: Markov Cluster Algorithm (MCL) method was used for mining the module of the network, and GO annotation for code genes of each module (with $\mathrm{p}<0.05$, the number of code gene of each mode $\geq 2$ ) [26].

\section{Quantitative RT-PCR}

Quantitative RT-PCR was used to verify the CCL type gene expression. The cDNA synthesis of these genes was prepared using First-Strand cDNA Synthesis Kit (Invitrogen) following the manufacturer's protocols. Then the RT-PCR was performed with SYBR Green Supermix (BioRad) according to the protocol as follows: (1) $95{ }^{\circ} \mathrm{C}$ for $2 \mathrm{~min}$; (2) 40 cycles of $95{ }^{\circ} \mathrm{C}$ for $15 \mathrm{~s}, 60{ }^{\circ} \mathrm{C}$ for $15 \mathrm{~s}$ and $72{ }^{\circ} \mathrm{C}$ for $15 \mathrm{~s}$; (3) melting curve analysis; (4) $50{ }^{\circ} \mathrm{C}$ for 30s; (4) All reactions were run in triplicate. These primers were synthesized by Life Technologies Company and the actin beta gene was used as control (Additional file 3: Table S1). The expression levels of these genes were measured by $\mathrm{Ct}$ value (threshhold cycle). And the relative expression level was calculated with the equation $2^{-\Delta \Delta C t}$.

\section{Results}

Inflammation and pathological injuries of the brain tissue of infected mice

The relative eosinophil count of white blood cells in peripheral blood of mice on 21st day post-infection with A.cantonensis was $2.67 \pm 0.58$, which was significantly higher than the control group $1.33 \pm 0.58(\mathrm{p}<0.05)$. HE staining showed that pathological injuries in brain parenchyma of the infected mice included hemorrhages, dilated vessels, necrosis and infiltration of a large amount of inflammatory cells (Fig. 1). These findings indicated that the infected mouse model was constructed successfully.

\section{Gene-expression analysis}

A total of $37.87 \mathrm{G}$ reads were generated from mouse brain tissue, of them 8.13G, 8.18G, 7.90G, 7.57G and 6.09G for post-infection of 2 days, 7 days, 14 days, 21 days and control groups respectively. After filtering out contaminating reads, a total of $149,556,822$ reads with an average map rate of $63.24 \%$ were produced from mouse brain cDNA libraries. The clean reads were assembled and blast with the reference genome using TopHat. Then Cufflinks generated a total of 24,942 transcripts.

The expression of IL-4 and IL-13 in brain tissue improved significantly at $14 \mathrm{dpi}$ and $21 \mathrm{dpi}$. IL-19, IL-10, IL-6, IL-27 and IL-5 also increased during late infection days. Besides these IL-1b, IL-15 and IL-1a also increased more than 5 fold at $21 \mathrm{dpi}$ (Table 1). The expression of ATF-3 improved significantly at late infection phase (Additional file 4: Table S2). 

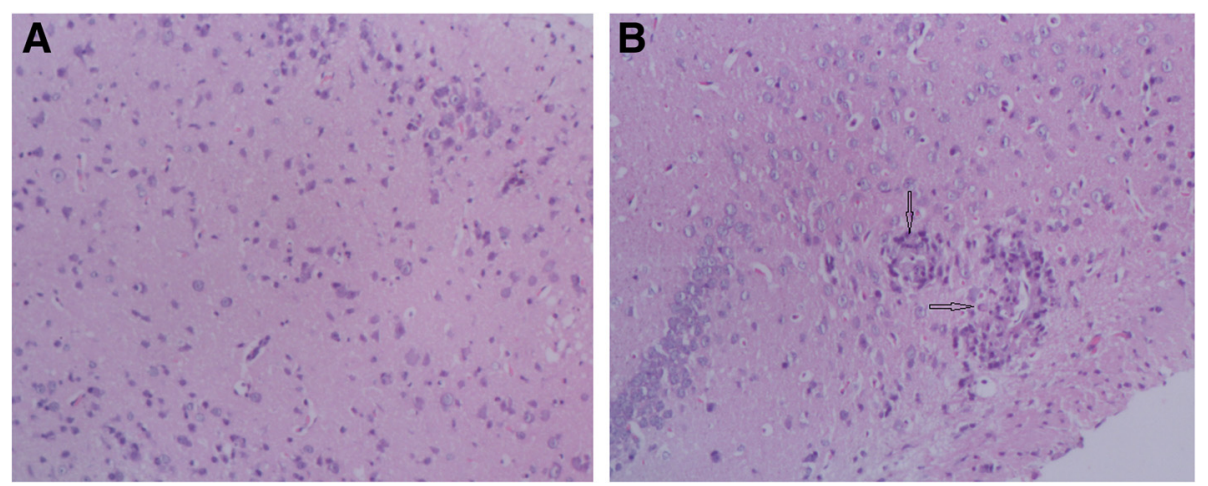

Fig. 1 HE staining of the brain tissues taken from control (a) and A. cantonensis infection group of 21 days (b). A: $\times 100$. B: $\times 100$, pathological injuries showing focal necrosis and infiltration of inflammatory cell in the brain tissue (showed by black star)

CCL2 (MIP-1) was not secreted in the control group, its expression improved at $2 \mathrm{dpi}$ and increased to the highest point at $21 \mathrm{dpi}$. CCL2 had the most significant change at $14 \mathrm{dpi}$ and $21 \mathrm{dpi}$ of the CC type chemokine expression data. It was followed by the change of expression of CCL8 at $14 \mathrm{dpi}$ and 21 dpi. CCL1 (I-309), CCL24

Table 1 Differential expression of interleukin. FPKM values of interleukin from brain tissue for different infection days (2 days, 7 days, 14 days and 21 days) in comparison with control group are listed

\begin{tabular}{|c|c|c|c|c|c|}
\hline Gene Symbol & Con & 2 days & 7 days & 14 days & 21 days \\
\hline$\| 10$ & 0 & 0 & 0 & 0.188 & 0.378 \\
\hline$\| 11$ & 0.247 & 0.182 & 0.353 & 0.437 & 0.458 \\
\hline$\| 13$ & 0 & 0 & 0 & 0.979 & 1.644 \\
\hline II15 & 0.042 & 0.223 & 0 & 0.618 & 0.319 \\
\hline$\| 16$ & 2.608 & 2.831 & 13.538 & 1.240 & 1.255 \\
\hline |l18 & 17.449 & 15.415 & 9.336 & 20.460 & 13.901 \\
\hline$\| 19$ & 0 & 0.042 & 0 & 0.044 & 0.746 \\
\hline Il1a & 0.151 & 0.358 & 0.058 & 1.640 & 0.904 \\
\hline$\| 1 b$ & 0.359 & 0.176 & 0.095 & 6.454 & 8.003 \\
\hline $111 f 5$ & 0 & 0 & 0.250 & 0 & 0 \\
\hline ||22 & 0.157 & 0.038 & 2.725 & 0.091 & 0.049 \\
\hline 1123a & 0.270 & 0.342 & 0.825 & 0.506 & 0.215 \\
\hline ||25 & 0.379 & 0.278 & 0.128 & 0.250 & 0.101 \\
\hline$\| 27$ & 0 & 0 & 0 & 0.081 & 0.295 \\
\hline 113 & 0 & 0 & 0 & 0.118 & 0.000 \\
\hline 1133 & 3.378 & 2.590 & 1.275 & 3.280 & 2.398 \\
\hline II34 & 18.909 & 18.201 & 11.212 & 14.285 & 20.597 \\
\hline$\| 4$ & 0 & 0 & 0 & 0.238 & 2.600 \\
\hline 115 & 0 & 0.024 & 0 & 0.178 & 0.276 \\
\hline 116 & 0 & 0 & 0 & 0.202 & 0.341 \\
\hline$\| f 2$ & 34.311 & 32.975 & 39.354 & 36.748 & 33.950 \\
\hline IIf3 & 5.945 & 4.882 & 5.886 & 4.881 & 4.977 \\
\hline
\end{tabular}

(Eotaxin-2), CCL11 (Eotaxin), CCL9 (MIP-3ß), CCL6, CCL7 (MCP-3), CCL12 and CCL4 (MIP-1 $\beta$ ) were increased more than 50 fold at $21 \mathrm{dpi}$ compared to the control group. CCL5 showed the highest value at $14 \mathrm{dpi}$ (Table 2).

Some lncRNAs showed significantly altered expression in infected brain tissues at the late phase of infection. H19 improved significantly at 14 dpi. Malat1 increased nearly 3 fold at late infection phase (Additional file 4: Table S2).

Table 2 Differential expression of CC type chemokines. FPKM values of CC type chemokines from five groups represented their expression level

\begin{tabular}{llllll}
\hline Gene Symbol & Con & 2 days & 7 days & 14 days & 21 days \\
\hline Cc121a & 0.277 & 0.049 & 0.709 & 1.077 & 0.463 \\
Ccl1 & 0 & 0 & 0 & 0.342 & 3.953 \\
Cc11 & 0 & 0 & 0 & 2.760 & 3.329 \\
Cc12 & 1.520 & 2.547 & 2.537 & 225.273 & 146.464 \\
Cc117 & 9.118 & 7.288 & 4.203 & 16.460 & 16.052 \\
Cc12 & 0 & 0.181 & 0.581 & 19.173 & 28.350 \\
Cc122 & 0.134 & 0.139 & 0.256 & 4.785 & 1.473 \\
Cc125 & 1.246 & 1.197 & 1.018 & 1.851 & 1.935 \\
Cc127a & 10.451 & 22.708 & 48.381 & 31.936 & 18.279 \\
Cc13 & 0.775 & 1.063 & 1.001 & 5.904 & 9.697 \\
Cc14 & 0.598 & 1.806 & 0.549 & 9.863 & 34.128 \\
Cc15 & 0.779 & 0.548 & 3.889 & 41.466 & 27.276 \\
Cc16 & 0.899 & 0.530 & 0.513 & 103.572 & 161.367 \\
Cc17 & 0.253 & 0 & 0 & 22.451 & 38.048 \\
Cc18 & 0.646 & 0.453 & 1.895 & 1021.010 & 1573.200 \\
Cc19 & 0.149 & 0.243 & 0.071 & 27.302 & 41.139 \\
Cc119 & 0.246 & 0.238 & 0.469 & 1.495 & 0.831 \\
Cc124 & 0.290 & 0.488 & 0.590 & 41.561 & 109.315 \\
Cc127b & 4.194 & 4.018 & 6.907 & 6.177 & 3.441 \\
Cc121b & 0.033 & 0.061 & 0.092 & 0.110 & 0.210 \\
\hline
\end{tabular}




\section{Quantitative RT-PCR}

To validate the deep sequencing data, the expression levels of CCL1, CCL2, CCL5, CCL7, CCL8, CCL11, CCL12, CCL24 were selected and quantified using Quantitative RT-PCR. The results showed that CCL1, 5, 7, 11, 24 secretion were increased with the development of CNS inflammatory progression. These $\mathrm{CC}$ type chemokines had relatively lower expression at $7 \mathrm{dpi}$, and increased to peak at $21 \mathrm{dpi}$. The expression level of CCL2 increased significantly at the early infection phase and reached the peak at $14 \mathrm{dpi}$ and still showed high expression at the late infection phase. CCL 8 and 12 were found to increase and
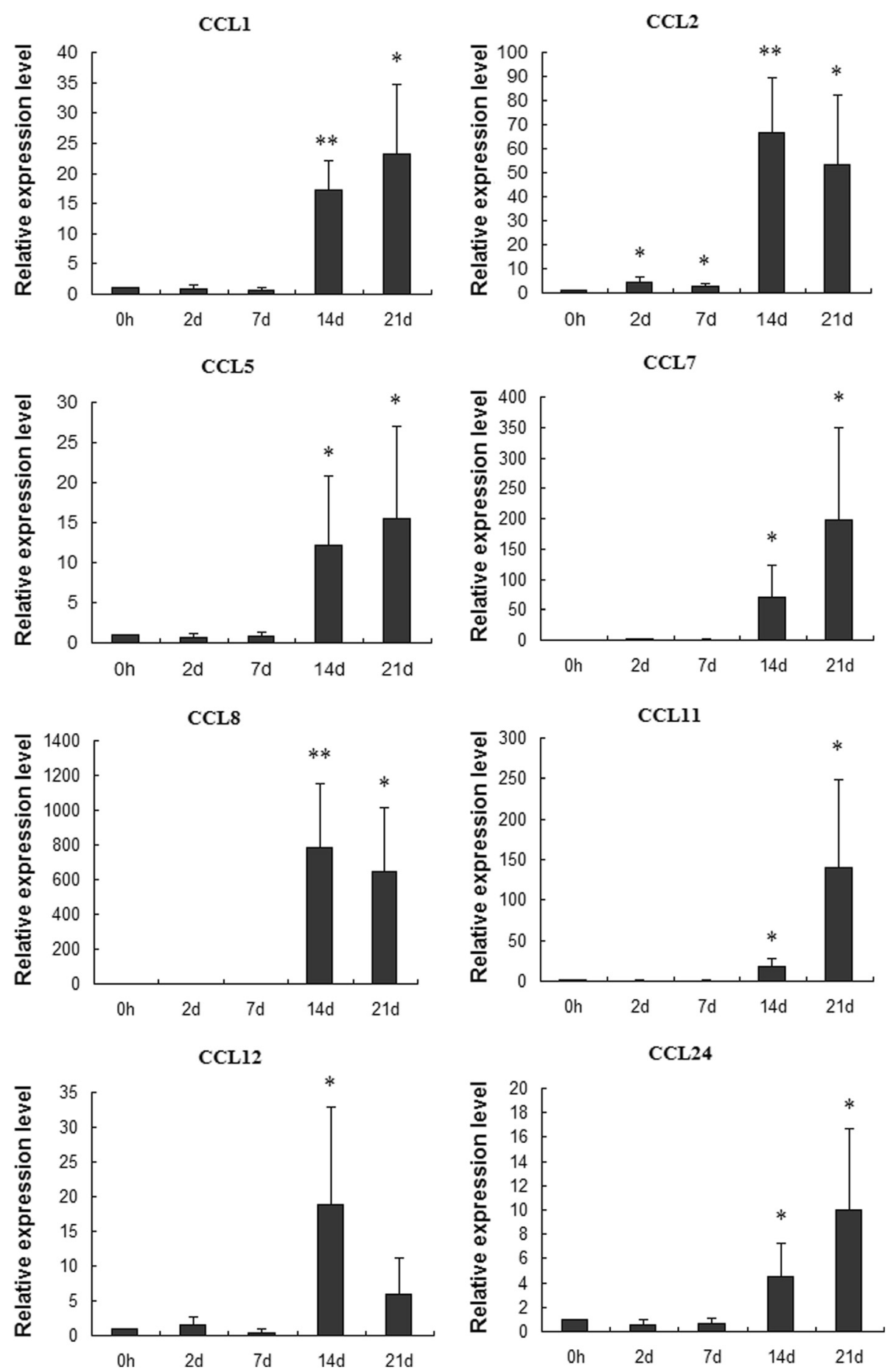

Fig. 2 Differential expression of CC chemokines validated by Q RT-PCR. The relative expression level was calculated with the equation 2 - $\Delta \Delta C \mathrm{ct}$ and the error bars represented the standard error $(n=5)$. * represents $p<0.05$, and **represents $p<0.01$ compared with control group 
reached the peak value at $14 \mathrm{dpi}$ and also had higher expression at $21 \mathrm{dpi}$ (Fig. 2). The results showed that these differentially expressed chemokines were involved in CNS inflammatory progression.

\section{LncRNA prediction}

Cufflinks regenerated transcripts. These transcripts were compared to previously annotated genes with Cuffcompare. Applying the screening model, a total of 1217 new lncRNA were obtained (Additional file 5: Table S3). The expression of these putative lncRNAs were analysed and it was shown that they were mainly distributed in chr2, chr11 and chr1 separately 158, 155 and 147 type (Additional file 6: Table S4, Table 3). These lncRNA candidates' data analyses will help to guide further IncRNA identifications and characterizations.

\section{Co-expression network}

Weighted gene co-expression network analysis identified 180 co-expression modules which include the number of code gene $\geq 8$. Ccl3, Ltb4r1, Cebpd, Mfng and Hp were highly and significantly correlated with the expression of CCL24. Gm4841, Clec4b1, Sirpb1a, Cd209d, Clec4d, Pira1, Egr1, Prg2 and Gm9733 were also highly and significantly correlated with the expression of CCL11. Significant correlation was shown in Zfp592, Wfdc17, Trem2, Cd44, Lpar5, Hcn1, Cbr2 and Irf4 with CCL8 (Fig. 3). The expression of CCL8 is correlated significantly with CCR8. The GO enrichment analysis from biological process of this module revealed that the two most enriched GO terms were myeloid cell differentiation and inflammatory response (Fig. 4). The co-expression network analysis showed that ATF-3 was highly and significantly correlated with the expression of Cebpd and Itgax (Additional file 7: Table S5).

Table 3 New IncRNA number of different chromosome

\begin{tabular}{ll}
\hline Chromosome & Num \\
\hline chr1 & 147 \\
chr10 & 75 \\
chr11 & 155 \\
chr12 & 95 \\
chr13 & 76 \\
chr14 & 76 \\
chr15 & 79 \\
chr16 & 93 \\
chr17 & 93 \\
chr18 & 79 \\
chr19 & 103 \\
chr2 & 63 \\
chr3 & 53 \\
\hline
\end{tabular}

\section{Discussion}

Our former research showed CCL8 and CCL11 in mouse brain samples increased gradually in parallel with the rising eosinophil counts in blood after infection [21]. In this study, these CC type chemokines (CCL1, 5, 7, 11, 24) normally had no or less secretion, however, they improved significantly at $14 \mathrm{dpi}$ and reached the top at $21 \mathrm{dpi}$. This phenomenon is consistent with the eosinophil change trend in cerebrospinal fluid (CSF) and peripheral blood. It has been shown that the worm recovery rate increased along with the infection time, highest at the third week in the infected mouse brain [27]. These chemokines serve as mediators for eosinophil recruitment in the development of CNS inflammation caused by $A$. cantonensis infection.

The expression of CCL2 also improved significantly during the early infection phase. It has been demonstrated that CCL2 could be induced in the brain during injury and trauma [28-30]. Research showed that the larvae migrated to the host brain tissue in the second day after infection. The movement of the larvae induced the injury of brain neurons [27]. The neurons and astrocyte can release the CCL2 in the pathogen stimulation [31]. Therefore, the invasion of $A$. cantonensis may induce some inflammatory factors such as CCL2 secretion in the brain at early infection. The involvement of CCL2 in neuropathic pain processing has been established in animal models [32, 33]. This up-regulation of CCL2 correlated with local monocyte and macrophage infiltration and pain processing $[34,35]$. CCL2 might play a major role in the neuropathic pain caused by $A$. cantonensis infection.

CCL8 showed significant changes at 14 dpi and $21 \mathrm{dpi}$. Module function analysis of co-expression network revealed that Lpar5 was highly and significantly correlated with the expression of CCL8. Lpar5 was one of the five known G protein-coupled receptors (GPCRs) [36]. GO analysis showed this module was mainly focused in the inflammatory response. In this study, CCR8 also increased at the late infection phase and its expression is correlated significantly with CCR8. CCR8-CCL8 is a newly identified chemokine receptor-ligand pair that mediates the skin accumulation of $\mathrm{TH} 2$ cells with the specific potential to drive chronic eosinophilic inflammation [37]. These data indicated that CCR8-CCL8 might be involved in recruitment of Th2 cells during the development of CNS inflammation caused by $A$. cantonensis infection.

These chemokines such as CCL2, 5, 7 and 8 played a key role in the specific recruitment of Th2 cells and eosinophils [38-40]. According to previous research, Th2-type cytokines may also stimulate recruitment of eosinophils and neutrophils through the release of CC (RANTES, MCP-3 and -4, eotaxin and eotaxin-2) and CXC chemokines [41]. In this current study, IL-4, IL-13, IL-19, IL-10, IL-6, IL-27 and IL-5 showed almost no 


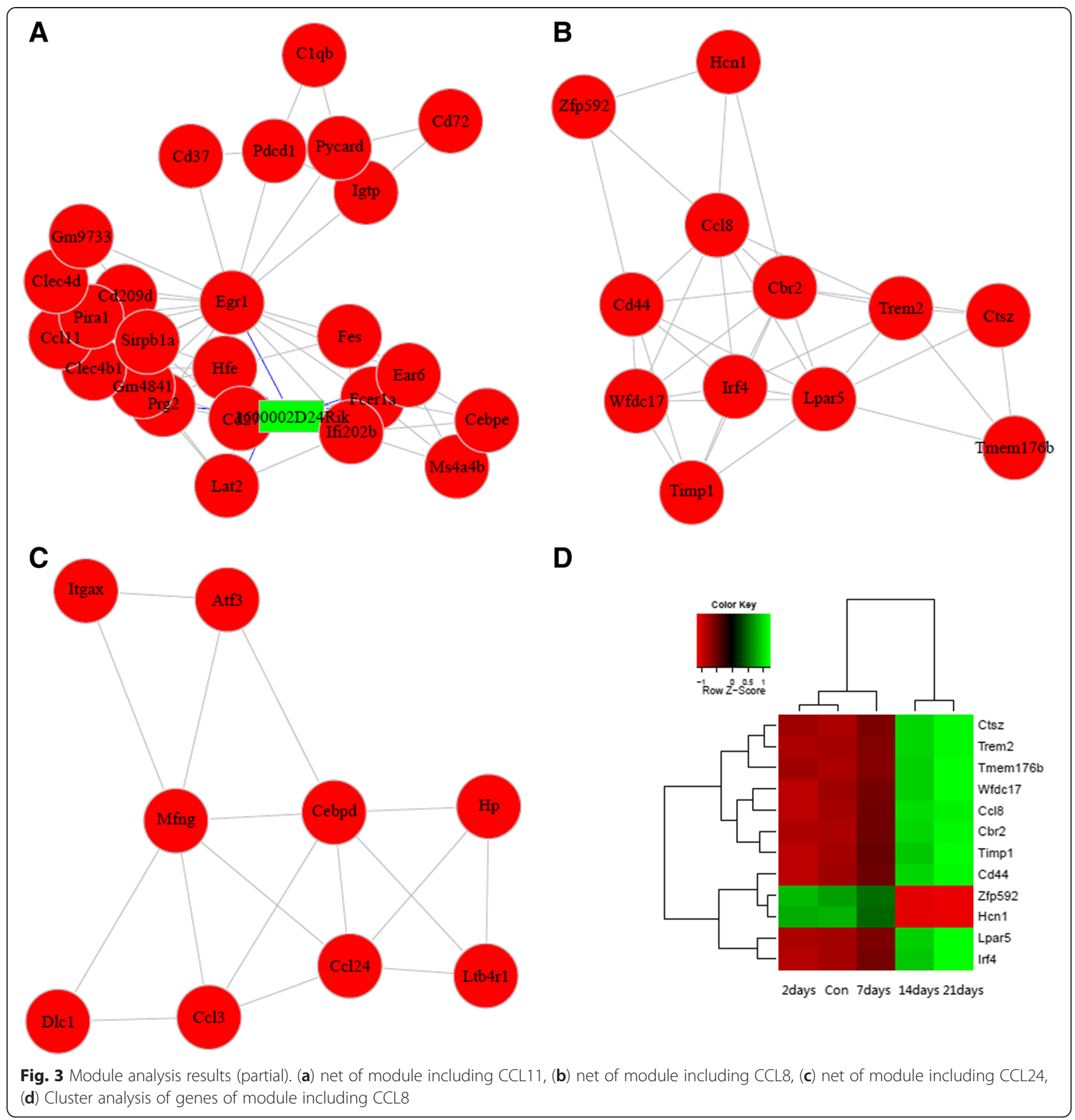

expression in the normal control group, whilst at 14 dpi and $21 \mathrm{dpi}$, IL-4 and IL-13 significantly increased, followed by IL-19, IL-10, IL-6, IL-27 and IL-5, which were highly expressed. The migration of the worm in the brain of host induced injury and inflammation of CNS [42, 43]. Macrophages, eosinophils and lymphocytes gathered at the site of inflammation and Th2-type cytokine secretion such as IL-4, IL-5, IL-10, and IL-13 were increased under the stimulation of worm antigen in the late infection phase. These might also promote the secretion of CC type chemokines. IL-5 plays a major role in the regulation of eosinophil formation, maturation, recruitment and survival [44]. Its improvement correlated with the trend of worm recovery in the brain of mice and the count of eosinophils, which also showed significant improvement both in peripheral blood and CSF at the late infection phase. IL-6 is primarily produced at sites of acute and chronic inflammation, it functions in inflammation and the maturation of B cells, and chronically elevated IL-6 levels lead to chronic 


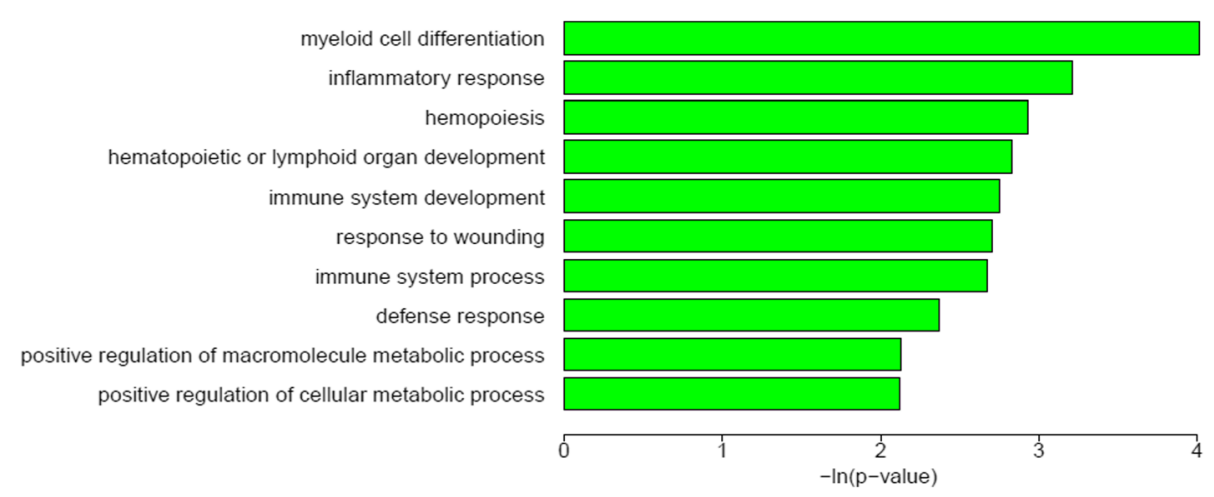

Fig. $4 \mathrm{GO}$ enrichment analysis (partial). GO enrichment analysis from biological process for the code genes of the module including CCL8

inflammation and fibrotic disorders [45]. IL-19 belongs to the IL-10 cytokine subfamily and it can lead to the activation of the signal transducer and activator of transcription 3 (STAT3) [46]. It was reported that it can lead to the higher expression of IL-6 and TNF-alpha and induce apoptosis which suggested it was involved in inflammatory responses and promoted the development of CNS inflammation [47-49]. There can be interplay between these different cytokines that can modulate cytokine levels and either up regulate or inhibit each other and function as a network with the specific potential to drive brain eosinophilic inflammation.

The pathological process induced by inflammatory factors in CNS has also been associated with changes in the transcription factor ATF-3, which plays a role in regulating axon growth and regeneration, and the induction of ATF-3 is linked with injury of the axon [50]. The pattern of its gradually increasing was in accordance with variation of the number change of worm body and pathological changes in the mouse brain. This indicated that the migration of the worm into the brain caused the injury of nerve cell and induced the high expression of ATF-3.

Recent studies have highlighted the roles of long noncoding RNAs (lncRNAs) in cancer, cardiovascular disease and other inflammation related diseases, and it is suggested that they might play critical roles in inflammation progression [51-53]. H19 genes are imprinted in mammals [54]; it showed different expression during late A. cantonensis infection. 1217 putative lncRNA were obtained in this study. According to the analysis by coexpression modules, it was shown that some lncRNAs was highly and significantly correlated with the expression of CCL24, CCL11and CCL8. Those A. cantonensis infection-related lncRNAs may provide potential novel therapeutic targets for A. cantonensis infection and other diseases. At present, we are preparing the animal and cell experiments to identify the checkpoint correlated to CCL8.

\section{Conclusions}

These cytokine networks play an important role in the development of CNS inflammation caused by A. cantonensis infection.

\section{Additional files}

Additional file 1: Figure S1. The overall flow of RNA library construction and deep sequencing.

Additional file 2: Figure S2. The overall flow of analysis of sequence data.

Additional file 3: Table S1. Primers of CC chemokines used in Q RT-PCR. Additional file 4: Table S2. All Refseq genes' expression.

Additional file 5: Table S3. New IncRNA information.

Additional file 6: Table S4. New IncRNA's expression.

Additional file 7: Table S5. The co-expression net-work.

\section{Competing interests}

The authors declare that they have no competing interests.

\section{Authors' contributions}

YL carried out the experiments and drafted the manuscript, WX and LQ participated in the sequence alignment and revised the manuscript. XL participated in the sequence alignment. ZY, LZ and ZX participated in the design of the study and performed the statistical analysis. WZ conceived of the study, and participated in its design and coordination and helped to draft the manuscript. WJ and LS performed the Q RT-PCR. All authors read and approved the final manuscript.

\section{Acknowledgments}

The authors would like to thank Beijing Berry Genomics Corparation for providing scientific assistance. This work was supported by the National Natural Science Foundation of China (grant no. 81271855,81261160324 and 81371836) and the grant from the National Basic Research Program of China (2010CB530004).

\section{Author details}

'Department of Preventive Medicine, School of Medicine, Three Gorges University, Yichang, China. ${ }^{2}$ Department of Parasitology, Zhongshan School of Medicine, Sun Yat-sen University, Guangzhou, China. ${ }^{3}$ Key Laboratory for Tropical Diseases Control, The Ministry of Education, Sun Yat-sen University, Guangzhou, China. ${ }^{4}$ Department of Preventive Medicine, School of Medicine, Ningbo University, Ningbo, China. ${ }^{5}$ Advanced Computing Research Laboratory, Institute of Computing Technology, Chinese Academy of Sciences, Beijing, China. 
Received: 22 January 2015 Accepted: 5 June 2015

\section{Published online: 14 June 2015}

\section{References}

1. Moreira VL, Giese EG, Melo FT, Simoes RO, Thiengo SC, Maldonado Jr A, et al. Endemic angiostrongyliasis in the Brazilian Amazon: natural parasitism of Angiostrongylus cantonensis in Rattus rattus and R. norvegicus, and sympatric giant African land snails, Achatina fulica. Acta Trop. 2013;125(1):90-7.

2. Eamsobhana P. Angiostrongyliasis in Thailand: epidemiology and laboratory investigations. Hawaii J Med Public Health. 2013;72(6 Suppl 2):28-32.

3. Lunn JA, Lee R, Smaller J, MacKay BM, King T, Hunt GB, et al. Twenty two cases of canine neural angiostronglyosis in eastern Australia (2002-2005) and a review of the literature. Parasit Vectors. 2012;5:70.

4. Deng ZH, Lv S, Lin JY, Lin RX, Pei FQ. An outbreak of angiostrongyliasis in Guanging, People's Republic of China: migrants vulnerable to an emerging disease. Southeast Asian J Trop Med Public Health. 2011;42(5):1047-53.

5. Evans-Gilbert T, Lindo JF, Henry S, Brown P, Christie CD. Severe eosinophilic meningitis owing to Angiostrongylus cantonensis in young Jamaican children: case report and literature review. Paediatr Int Child Health. 2014;34(2):148-52.

6. Padilla-Docal B, Iglesias-Gonzalez I, Bu-Coifiu-Fanego R, Socarras-Hernandez CA, Dorta-Contreras AJ. Intrathecal activation as a typical immune response within the central nervous system in angiostrongyliasis. Am J Trop Med Hyg. 2013;88(2):230-5.

7. Slom T, Johnson S. Eosinophilic Meningitis. Curr Infect Dis Rep. 2003;5(4):322-8.

8. Wiwanitkit S, Wiwanitkit V. Eosinophilic meningitis due to Angiostrongylus cantonensis with confirmed finding of intrathecal parasite. Arq Neuropsiquiatr. 2014;72(3):261.

9. Morassutti AL, Thiengo SC, Fernandez M, Sawanyawisuth K, Graeff-Teixeira C. Eosinophilic meningitis caused by Angiostrongylus cantonensis: an emergent disease in Brazil. Mem Inst Oswaldo Cruz. 2014;109(4):399-407.

10. Liu LH, He HJ, Lv ZY, Wei J, Zeng X, Liang JY, et al. The mRNA level of the galectin-10 of Angiostrongylus cantonensis induced by reactive oxygen stress. Parasitol Res. 2013;112(3):933-43.

11. Intapan PM, Kittimongkolma S, Niwattayakul K, Sawanyawisuth K, Maleewong W. Cerebrospinal fluid cytokine responses in human eosinophilic meningitis associated with angiostrongyliasis. J Neurol Sci. 2008;267(1-2):17-21.

12. LV S, Zhang Y, Chen SR, Wang LB, Fang W, Chen F, et al. Human angiostrongyliasis outbreak in Dali China. PLoS Negl Trop Dis. 2009;3(9), e520.

13. Oehler E, Ghawche F, Delattre A, Berberian A, Levy M, Valour F. Angiostrongylus cantonensis eosinophilic meningitis: a clinical study of 42 consecutive cases in French Polynesia. Parasitol Int. 2014;63(3):544-9.

14. Iwata T, Fujii T, Morii K, Saito M, Sugiyama J, Nishio H, et al. Cytokine profile in cervical mucosa of Japanese patients with cervical intraepithelial neoplasia. Int J Clin Oncol. 2014;20(1):126-33.

15. Teixeira MM, Wells TN, Lukacs NW, Proudfoot AE, Kunkel SL, Williams TJ, et al. Chemokine-induced eosinophil recruitment: Evidence of a role for endogenous eotaxin in an in vivo allergy model in mouse skin. J Clin Invest. 1997;100(7):1657-66.

16. Lukacs NW, Standiford TJ, Chensue SW, Kunkel RG, Strieter RM, Kunkel SL. C-C chemokine-induced eosinophil chemotaxis during allergic airway inflammation. J Leukoc Biol. 1996;60(5):573-8.

17. Lukacs NW, Prosser DM, Wiekowski M, Lira SA, Cook DN. Requirement for the chemokine receptor CCR6 in allergic pulmonary inflammation. J Exp Med. 2001:194(4):551-5.

18. Gerard C, Rollins BJ. Chemokines and disease. Nat Immunol. 2001;2(2):108-15.

19. Ying S, Robinson DS, Meng Q, Barata LT, McEuen AR, Buckley MG, et al. C-C chemokines in allergen-induced late-phase cutaneous responses in atopic subjects: association of eotaxin with early 6-hour eosinophils, and of eotaxin-2 and monocyte chemoattractant protein-4 with the later 24-hour tissue eosinophilia, and relationship to basophils and other $\mathrm{C}-\mathrm{C}$ chemokines (monocyte chemoattractant protein-3 and RANTES). J Immunol. 1999;163(7):3976-84.

20. Chang EE, Chung LY, Yen CM. Kinetics of change in the eotaxin concentration in serum and cerebrospinal fluid of mice infected with Angiostrongylus cantonensis. Parasitol Res. 2004;92(2):137-41.
21. Li S, Yang F, Ji P, Zeng X, Wu X, Wei J, et al. Eosinophil chemotactic chemokine profilings of the brain from permissive and non-permissive hosts infected with Angiostrongylus cantonenis. Parasitol Res. 2014;113(2):517-25.

22. Feng $Y$, Zeng $X$, Li WH, Wang WC, Ou-Yang LS, Sun $X$, et al. Animal model of human disease with optic neuritis: neuropapillitis in a rat model infected with Angiostrongylus cantonensis. Parasitol Res. 2014;113(11):4005-13.

23. Yu L, Liao Q, Zeng X, Lv Z, Zheng H, Zhao Y, et al. MicroRNA expressions associated with eosinophilic meningitis caused by Angiostrongylus cantonensis infection in a mouse model. Eur J Clin Microbiol Infect Dis. 2014;33(8):1457-65.

24. Bauer BK, Isom SC, Spate LD, Whitworth KM, Spollen WG, Blake SM, et al. Transcriptional profiling by deep sequencing identifies differences in mRNA transcript abundance in in vivo-derived versus in vitro-cultured porcine blastocyst stage embryos. Biol Reprod. 2010;83(5):791-8.

25. Zhao Y, Luo H, Chen X, Xiao Y, Chen R. Computational methods to predict long noncoding RNA functions based on co-expression network. Methods Mol Biol. 2014;1182:209-18.

26. Liao Q, Liu C, Yuan X, Kang S, Miao R, Xiao H, et al. Large-scale prediction of long non-coding RNA functions in a coding-non-coding gene co-expression network. Nucleic Acids Res. 2011;39(9):3864-78.

27. OuYang L, Wei J, Wu Z, Zeng X, Li Y, Jia Y, et al. Differences of larval development and pathological changes in permissive and nonpermissive rodent hosts for Angiostrongylus cantonensis infection. Parasitol Res. 2012;111(4):1547-57.

28. Van Steenwinckel J, Auvynet C, Sapienza A, Reaux-Le Goazigo A, Combadiere C, Melik PS. Stromal cell-derived CCL2 drives neuropathic pain states through myeloid cell infiltration in injured nerve. Brain Behav Immun. 2015;45:198-210.

29. Guo YQ, Zheng LN, Wei JF, Hou XL, Yu SZ, Zhang WW, et al. Expression of CCL2 and CCR2 in the hippocampus and the interventional roles of propofol in rat cerebral ischemia/reperfusion. Exp Ther Med. 2014;8(2):657-61.

30. Yao Y, Tsirka SE. Monocyte chemoattractant protein-1 and the blood-brain barrier. Cell Mol Life Sci. 2014;71(4):683-97.

31. Peterson KE, Errett JS, Wei T, Dimcheff DE, Ransohoff R, Kuziel WA, et al. MCP-1 and CCR2 contribute to non-lymphocyte-mediated brain disease induced by Fr98 polytropic retrovirus infection in mice: role for astrocytes in retroviral neuropathogenesis. J Virol. 2004;78(12):6449-58.

32. Tanaka T, Minami M, Nakagawa T, Satoh M. Enhanced production of monocyte chemoattractant protein-1 in the dorsal root ganglia in a rat model of neuropathic pain: possible involvement in the development of neuropathic pain. Neurosci Res. 2004;48(4):463-9.

33. Graber DJ, Costine BA, Hickey WF. Early inflammatory mediator gene expression in two models of traumatic brain injury: ex vivo cortical slice in mice and in vivo cortical impact in piglets. J Neuroinflammation. 2015;12(1):76.

34. Hammond MD, Taylor RA, Mullen MT, Ai Y, Aguila HL, Mack M, et al. CCR2+ Ly6C (hi) inflammatory monocyte recruitment exacerbates acute disability following intracerebral hemorrhage. J Neurosci. 2014;34(11):3901-9.

35. Bose $\mathrm{S}, \mathrm{Cho} \mathrm{J}$. Role of chemokine CCL2 and its receptor CCR2 in neurodegenerative diseases. Arch Pharm Res. 2013;36(9):1039-50.

36. Choi JW, Herr DR, Noguchi K, Yung YC, Lee CW, Mutoh T, et al. LPA receptors: subtypes and biological actions. Annu Rev Pharmacol Toxicol. 2010;50:157-86.

37. Islam SA, Chang DS, Colvin RA, Byrne MH, McCully ML, Moser B, et al. Mouse CCL8, a CCR8 agonist, promotes atopic dermatitis by recruiting IL-5+ T (H)2 cells. Nat Immunol. 2011;12(2):167-77.

38. Lee YG, Jeong JJ, Nyenhuis S, Berdyshev E, Chung S, Ranjan R, et al. Recruited alveolar macrophages, in response to airway epithelial-derived MCP-1/CCL2, regulate airway inflammation and remodeling in allergic asthma. Am J Respir Cell Mol Biol. 2014;52(6):772-84.

39. Suzuki Y, Asano K, Niimi K, Miyata J, Shiraishi Y, Fukunaga K, et al. TP receptor-mediated release of eosinophil chemotactic activity from human bronchial smooth muscle cells. Eur J Pharmacol. 2008;600(1-3):133-9.

40. de Nadai P, Chenivesse C, Gilet J, Porte H, Vorng H, Chang Y, et al. CCR5 usage by CCL5 induces a selective leukocyte recruitment in human skin xenografts in vivo. J Invest Dermatol. 2006;126(9):2057-64.

41. Meyer-Hoffert U, Lezcano-Meza D, Bartels J, Montes-Vizuet AR, Schroder JM, Teran LM. Th2- and to a lesser extent Th1-type cytokines upregulate the production of both CXC (IL-8 and gro-alpha) and CC (RANTES, eotaxin, eotaxin-2, MCP-3 and MCP-4) chemokines in human airway epithelial cells. Int Arch Allergy Immunol. 2003;131(4):264-71.

42. Feng $Y$, Zeng $X$, Li WH, Wang WC, Chen W, Ou-Yang LS, et al. The pathogenesis of optic neuritis caused by Angiostrongylus cantonensis in BALB/C mice. Parasit Vectors. 2014;7:339. 
43. Tsai HC, Lee BY, Yen CM, Wann SR, Lee SS, Chen YS. Dexamethasone inhibits brain apoptosis in mice with eosinophilic meningitis caused by Angiostrongylus cantonensis infection. Parasit Vectors. 2015;8(1):200.

44. Garcia G, Taille C, Laveneziana P, Bourdin A, Chanez P, Humbert M. Anti-interleukin-5 therapy in severe asthma. Eur Respir Rev. 2013;22(129):251-7.

45. Fontes JA, Rose NR, Cihakova D. The varying faces of IL-6: From cardiac protection to cardiac failure. Cytokine. 2015;74(1):62-8.

46. Kunz S, Wolk K, Witte E, Witte K, Doecke WD, Volk HD, et al. Interleukin (IL)-19, IL-20 and IL-24 are produced by and act on keratinocytes and are distinct from classical ILs. Exp Dermatol. 2006;15(12):991-1004.

47. Azuma YT, Matsuo Y, Kuwamura M, Yancopoulos GD, Valenzuela DM, Murphy AJ, et al. Interleukin-19 protects mice from innate-mediated colonic inflammation. Inflamm Bowel Dis. 2010;16(6):1017-28.

48. Hsu YH, Li HH, Sung JM, Chen WT, Hou YC, Chang MS. Interleukin-19 mediates tissue damage in murine ischemic acute kidney injury. PLoS One. 2013;8(2), e56028.

49. Liao YC, Liang WG, Chen FW, Hsu JH, Yang JJ, Chang MS. IL-19 induces production of IL-6 and TNF-alpha and results in cell apoptosis through TNF-alpha. J Immunol. 2002;169(8):4288-97.

50. Stenberg L, Kanje M, Dolezal K, Dahlin LB. Expression of activating transcription factor 3 (ATF 3) and caspase 3 in Schwann cells and axonal outgrowth after sciatic nerve repair in diabetic BB rats. Neurosci Lett. 2012;515(1):34-8.

51. Cai B, Song XQ, Cai JP, Zhang S. HOTAIR: acancer-related long non-coding RNA. Neoplasma. 2014;61(4):379-91.

52. Leung A, Trac C, Jin W, Lanting L, Akbany A, Saetrom $P$, et al. Novel long noncoding RNAs are regulated by angiotensin II in vascular smooth muscle cells. Circ Res. 2013;113(3):266-78.

53. Deng K, Guo X, Wang H, Xia J. The IncRNA-MYC regulatory network in cancer. Tumour Biol. 2014;35(10):9497-503.

54. Tran VG, Court F, Duputie A, Antoine E, Aptel N, Milligan L, et al. H19 antisense RNA can up-regulate lgf2 transcription by activation of a novel promoter in mouse myoblasts. PLoS One. 2012;7(5), e37923.

\section{Submit your next manuscript to BioMed Central and take full advantage of:}

- Convenient online submission

- Thorough peer review

- No space constraints or color figure charges

- Immediate publication on acceptance

- Inclusion in PubMed, CAS, Scopus and Google Scholar

- Research which is freely available for redistribution 